\section{Transportzeiten von diagnostischen Proben}

W. G. Guder

München, Deutschland

Synonym(e) Präanalytische Zeit außerhalb des Labors

Englischer Begriff transport time

Definition Als Transportzeit ( $\triangleright$ Turnaround-Time, TAT) wird die Zeit definiert, die eine diagnostische Probe vom Verlassen des Ortes der Probengewinnung bis zur Registrierung im Labor benötigt.

Beschreibung Im Zusammenhang mit der Erfassung der einzelnen Zeiten des diagnostischen Prozesses wurde die präanalytische Zeit außerhalb des Labors mit 15-20\% als die größte innerhalb des gesamten Prozesses der Diagnostik erkannt. Dieser prozentuelle Anteil setzt optimale Organisation und Labornähe voraus, da sonst wesentlich größere Transportzeiten zu erwarten sind. Die Transportzeit beeinflusst das zu erwartende $\triangleright$ Messergebnis, wenn sie die Zeit der Stabilität des Analyten in der Matrix übersteigt, wenn während des Transports mechanische oder physikalisch chemische Vorgänge die Zusammensetzung der Matrix verän- dern (z. B. mechanische Hämolyse durch zu heftige Schüttelvorgänge in Rohrpostanlagen, Einfrieren der Blutprobe mit Erzeugung einer $>$ Hämolyse bei Durchmessen kalter Zonen während des Transports, Überhitzung der Probe durch unsachgemäße Aufbewahrung während des Transports) oder metabolische Vorgänge in der Probe den zu messenden Analyten vermehren (z. B. \ Laktat, Ammoniak) oder vermindern (z. B. - Glukose oder $p \mathrm{CO}_{2}$ ). Daher ist es anzustreben, dass für jeden Analyten die Form der Probe (z. B. Plasma oder Vollblut) und die Temperatur und Zeit während des Transports dem Absender bekannt ist, um entsprechende Maßnahmen zur Vermeidung falscher Ergebnisse zu ergreifen. Es wurden internationale Empfehlungen zur Definition der maximalen Stabilität jedes Analyten veröffentlicht und Maßnahmen zur Dokumentation der präanalytischen Zeiten empfohlen.

\section{Literatur}

Guder WG, Fiedler GM, da Fonsecca-Wollheim F, Schmitt Y, Töpfer G, Wisser H, Zawta B (2015) Quality of diagnostic samples. BD-Diagnostics, Oxford

World Health Organization (WHO) (1977) Guidance on regulation for transport of infectious substances. WHO, Geneva 